\title{
Serotyping of Streptococcus pneumoniae strains by coagglutination
}

\author{
LE SMART \\ From the Department of Bacteriology, Stobhill Hospital, Glasgow, Scotland
}

SUMMARY The coagglutination test, which uses staphylococcal protein A, for serotyping strains of Streptococcus pneumoniae, was extended to include serotyping within serogroups. Serotyping was performed with "factor sera" prepared in the laboratory. Fifty one strains of $S$ pneumoniae, which belonged to one of the seven serogroups included in the 14 valent vaccine formulation, were tested, and no inconsistency was found when compared with the capsule swelling reaction.

Serotyping of Streptococcus pneumoniae is a valuable epidemiological tool and provides information about the potential of available pneumococcal vaccines (Pneumovax and Pneumovax-2) for protecting subjects from specific pneumococcal infections. It is not known whether vaccination with a given serotype protects against infections by related types being contained within the same serogroup. The distribution of serotypes associated with infection varies geographically and according to the age of the patient, ${ }^{1}$ and it is therefore important to have data on incidence of serotype and distribution.

Most isolates of $S$ pneumoniae in man are capsulate. Differences in the composition and structure of the capsular material allow 83 serotypes to be distinguished. In the Danish nomenclature, now universally accepted, there are 27 monotypes and 19 serogroups. The serogroups consist of between two and four types that possess common antigenic determinants but which can be distinguished serologically. The 19 serogroups are thus divided into 56 serotypes using specially prepared "factor sera" that are rendered monospecific by absorption. ${ }^{2}$

Typing of pneumococci by means of the capsule swelling reaction, ${ }^{3}$ counterimmunoelectrophoresis, ${ }^{4}$ and coagglutination, ${ }^{5}$ with commercially available pneumococcal antisera can identify strains belonging to one of the 27 monotypes or 19 serogroups. Serotyping within serogroups, however, requires specially prepared "factor sera," which are not commercially available. Henrichsen ${ }^{6}$ compared the capsule swelling reaction with counterimmunoelectrophoresis for serotyping pneumococci and for serotyping within serogroups. He found that the capsule swelling reac-

Accepted for publication 2 December 1985 tion was preferable. Coagglutination, however, is an alternative technique to capsule swelling for serotyping and correlates well with that technique. ${ }^{5}$ Furthermore, coagglutination is rapid and economical. ${ }^{7}$ This paper describes the extension of the coagglutination test to serotyping within serogroups using specially prepared "factor sera."

\section{Material and methods}

\section{Reference strains of $S$ pneumoniae}

Concentrated suspensions for the preparation of antisera and for absorption (see below) were prepared from clinical isolates that had been serotyped, or supplied as lyophilised cultures by Dr J Henrichsen, Streptococcus Department, Statens Seruminstitut, Copenhagen.

\section{PREPARATION OF STOCK PNEUMOCOCCAL SUSPENSIONS}

The growth from two blood agar plates (incubated anaerobically with $5-10 \%$ carbon dioxide for 18 hours) was used to inoculate $500 \mathrm{ml}$ of Todd Hewitt broth (Difco 0492-01) containing 5\% horse serum (Gibco Europe 034-6050). Cultures were incubated at $37^{\circ} \mathrm{C}$ for four to seven hours aerobically until growth was in the late log phase. Subculture was carried out to confirm purity, and $2 \%(\mathrm{vol} / \mathrm{vol})$ formaldehyde was added. The suspensions were left overnight at room temperature. The bacterial cells were then harvested by centrifugation ( $3500 \mathrm{rpm}$ for 30 minutes), and washed twice with phosphate buffered saline (PBS, pH 7.2) containing $0.5 \%$ formaldehyde. Concentrated suspension $(10 \mu \mathrm{l})$ was resuspended to $1 \mathrm{ml}$ in phosphate buffered saline and filmed to confirm that all the bacteria were Gram positive and possessed 
a well developed capsule. Stock suspensions were stored at $4^{\circ} \mathrm{C}$.

\section{PNEUMOCOCCAL ANTISERA}

Diagnostic pneumococcal antisera were obtained from the Statens Seruminstitut, Copenhagen. Antisera to types $6 \mathrm{~A}, 6 \mathrm{~B}, 7 \mathrm{~F}, 7 \mathrm{~A}, 7 \mathrm{~B}, 7 \mathrm{C}, 9 \mathrm{~N}, 9 \mathrm{~A}, 9 \mathrm{~V}, 9 \mathrm{~L}$, $12 \mathrm{~F}, 12 \mathrm{~A}, 18 \mathrm{~F}, 18 \mathrm{~A}, 18 \mathrm{~B}, 18 \mathrm{C}, 19 \mathrm{~F}, 19 \mathrm{~A}, 19 \mathrm{C}, 23 \mathrm{~F}$, $23 \mathrm{~A}$, and 28 were raised in rabbits using inocula prepared from stock pneumococcal suspensions diluted in phosphate buffered saline to give 1-2 $\times 10^{9}$ diplococci/ml (Lund and Henrichsen. ${ }^{3}$ )

\section{PREPARATION OF STABILISED STAPHYLOCOCCI (PROTEIN A)}

Staphylococcus aureus Cowan 1 (NCTC 8530) was grown on plates of CCY agar (Woodin modification), overlaid with dialysis membrane (Technicon part number 933-0225-01), and incubated at $37^{\circ} \mathrm{C}$ overnight. The bacteria were harvested from the membranes by agitation in phosphate buffered saline, the suspension washed twice in phosphate buffered saline, and suspended in $0.5 \%$ formaldehyde in phosphate buffered saline for two hours at room temperature. The formolinised suspension was washed twice in phosphate buffered saline, heated at $80^{\circ} \mathrm{C}$ for one hour, ${ }^{5}$ washed a further three times in phosphate buffered saline, and resuspended at a concentration of $10 \%(\mathrm{vol} / \mathrm{vol})$ in phosphate buffered saline containing $0 \cdot 1 \%$ sodium azide.

\section{PRODUCTION OF FACTOR SERA}

Antisera to types 6A, 6B, 7F, 7A, 7B, 7C, 9N, 9A, 9V, 9L, 12F, 12A, 18F, 18A, 18B, 18C, 19A, 19F, 19C, $23 \mathrm{~A}$, and $23 \mathrm{~F}$ were absorbed with formolinised suspensions of the appropriate heterologous strains to remove common serogroup antibodies and to produce factor sera ${ }^{2}$ as follows. Formolinised suspension $(500 \mu \mathrm{l})$ was centrifuged at $3500 \mathrm{rpm}$ for 30 minutes, and the supernatant fluid removed. Antiserum $(250 \mu \mathrm{l})$ was absorbed by resuspending the bacterial deposit in the serum at room temperature for 15-30 minutes. A second absorption was carried out in the same way using $500 \mu \mathrm{l}$ of the same suspension. Absorbed serum $(50 \mu \mathrm{l})$ was mixed with $500 \mu \mathrm{l}$ of protein $A$ and tested against appropriate suspensions of serotypes to confirm complete removal of homologous antibody. Although two absorptions were generally sufficient to remove all homologous antibody, some high titre antisera required a further absorption.

\section{COAGGLUTINATION}

Fifty microlitres of each antiserum (Pools A-I) serogroup sera and monotypic sera (Statens Seruminstitut, Copenhagen) were added to $500 \mu \mathrm{l}$ of stabilised staphylococcal suspension. These reagents were used for serotyping clinical isolates of $S$ pneumoniae as follows. Ten to 20 colonies of $S$ pneumoniae from an overnight anaerobic culture on blood agar were emulsified in $0.5 \mathrm{ml}$ of phosphate buffered saline. Tests were performed on 3 by 1 inch glass slides by mixing $1 \mu \mathrm{l}$ of bacterial suspension with $1 \mu \mathrm{l}$ of each of nine coagglutination reagents (Pool A-I, Statens Seruminstitut). The individual monotype or serogroup was determined by testing with single monotype or serogroup reagents appropriate to the pool that gave the positive reaction. The reactions were read by detecting visible clumping after rocking the slide to and fro for two minutes. Only strong reactions appearing within the two minutes were considered to be specific for pneumococcal capsular polysaccharide. In most instances positive reactions were detected in a few seconds.

Serotyping within the seven serogroups $(6,7,9,12$, 18,19 , and 23 ) represented in the 14 valent vaccine was performed using coagglutination reagents prepared from factor sera. The serotype was determined from the results of the coagglutination test when each strain was tested with each factor serum for the serogroup, according to the typing scheme set out by Lund and Henrichsen. ${ }^{3}$

\section{Results}

Table 1 shows the results of the coagglutination test with factor sera performed on stock reference strains of $S$ pneumoniae. The reactions observed with factor sera adsorbed to protein A agreed with the reactions given by capsule swelling (Lund and Henrichsen ${ }^{3}$ ).

Table 2 details the results of the coagglutination test and the capsule swelling reaction performed on 51 clinical isolates of $S$ pneumoniae, which belonged to one of the seven serogroups in the 14 valent vaccine. For clinical isolates the results of serotyping within serogroups using appropriate factor sera adsorbed to staphylococcal protein A agreed with the results obtained by capsule swelling.

\section{Discussion}

The results show that factor sera adsorbed to staphylococcal protein A can accurately serotype strains of $S$ pneumoniae by coagglutination. Although only a limited number of serotypes within serogroups are reported here, the results of the coagglutination test and capsule swelling were in complete agreement, and there is no reason to suspect that the results obtained were atypical.

Serotyping is an accurate and precise method for identifying isolates of $S$ pneumoniae: and it permits definitive identification $24-48$ hours earlier than is possible by current procedures for presumptive 
Table 1 Coagglutination results of reference strains

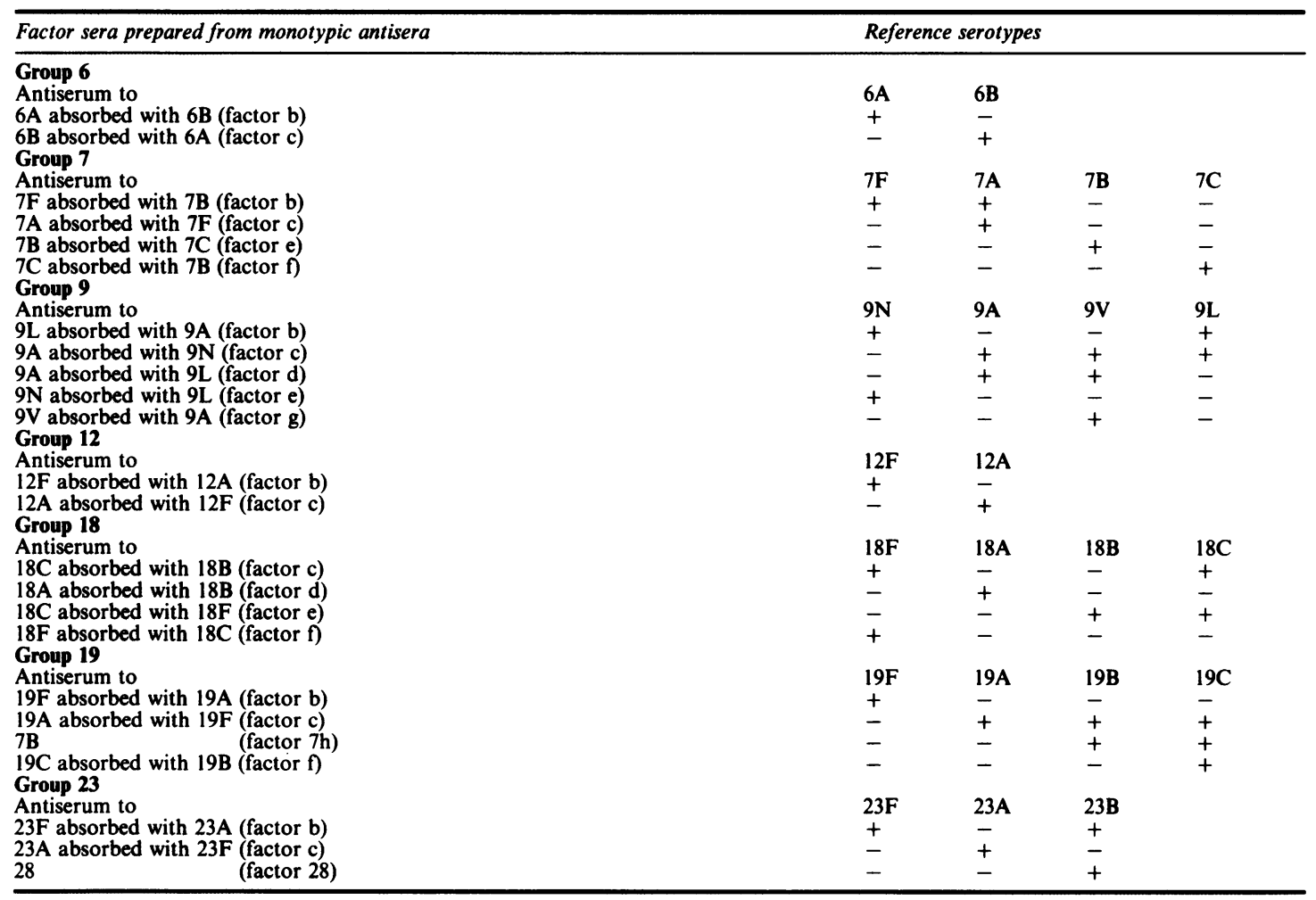

identification, such as optochin susceptibility. Determination of serotype also helps interpret the clinical importance of pneumococcal isolates, particularly from respiratory specimens, as the lower numbered serotypes have a greater potential for systemic disease. $^{4}$

Serotyping is, of course, necessary for epidemiological studies, ${ }^{48}$ and the investigation of pneu-

Table 2 Comparison of capsule swelling reaction and coagglutination test for serotyping strains of $S$ pneumoniae

\begin{tabular}{lll}
\hline Serotype & Capsule swelling & Coagglutination \\
\hline 6A & 3 strains & 3 strains \\
6B & 8 strains & 8 strains \\
$7 \mathrm{~F}$ & 7 strains & 7 strains \\
$7 \mathrm{C}$ & 1 strain & 1 strain \\
$9 \mathrm{~N}$ & 4 strains & 4 strains \\
$9 \mathrm{~A}$ & 2 strains & 2 strains \\
$9 \mathrm{~V}$ & 3 strains & 3 strains \\
12F & 2 strains & 2 strains \\
18A & 1 strain & 1 strain \\
18C & 4 strains & 4 strains \\
19F & 7 strains & 7 strains \\
19A & 4 strains & 4 strains \\
19C & 1 strain & 1 strain \\
23F & 4 strains & 4 strains \\
Total & 51 & 51 concordance $=100 \%$ \\
\hline
\end{tabular}

mococcal cross infection in hospital. ${ }^{9}$ Broome et al ${ }^{10}$ suggested that the efficiency of the vaccine can be assessed by comparing the incidence of type specific disease in vaccinated and non-vaccinated subjects. Routine serotyping, however, is rarely performed in clinical laboratories, and in the small number where it is practised the data produced are often incomplete, as not all the sera necessary for serotyping within serogroups are available. The technique used in reference laboratories for serotyping pneumococci is the capsule swelling reaction, but most clinical laboratories have neither the personnel with the necessary expertise nor the funds to adopt the method routinely.

Coagglutination is just as accurate as the capsule swelling reaction for serotyping pneumococci. As the test is based on a slide agglutination procedure it should be more acceptable to routine laboratories: no special training or equipment is required. Furthermore, this study shows that coagglutination can be adapted for serotyping within serogroups, a test at present only performed in specialised centres. In the method described here $5 \mu \mathrm{l}$ of antiserum was sufficient to perform 50 tests at a cost of $10 \mathrm{p}$ per isolate. The technique can also be used to determine the serotype 
of pneumococcal antigen in clinical material, and, as has been shown previously, ${ }^{11}$ gives a more accurate assessment of serotype prevalence than that obtained by culture alone.

I thank Professor MC Timbury and Dr DJ Platt, University Department of Bacteriology, Glasgow Royal Infirmary for helpful advice and criticism while preparing this manuscript, and Dr J Henrichsen, Streptococcus Department, Statens Seruminstitut, Copenhagen for serotyping the strains by capsule swelling.

\section{References}

${ }^{1}$ Austrian R. Some observations on the Pneumococcus and on the current status of pneumococcal diseases and its prevention. Rev Infect Dis 1981;3 (Suppl):S1-17.

${ }^{2}$ Henrichsen J. The pneumococcal typing system and pneumococcal surveillance. $J$ Infect 1979;1 (Suppl 2):31-7.

${ }^{3}$ Lund E, Henrichsen J. Laboratory diagnosis, serology and epidemiology of Streptococcus pneumoniae. In: Bergan T, Norris JR, eds. Methods in microbiology 12. New York: Academic Press, 1978:241-62.

${ }^{4}$ Sottile MI, Rytel MW. Application of counterimmunoelectrophoresis in the identification of Streptococcus pneumoniae in clinical isolates. J Clin Microbiol 1975;2:173-7.

${ }^{5}$ Kronvall G. A rapid slide-agglutination method for typing pneumococci by means of specific antibody adsorbed to protein Acontaining staphylococci. J Med Microbiol 1973;6:187-90.

${ }^{6}$ Henrichsen J, Berntsson E, Kaijser B. Comparison of counterimmunoelectrophoresis and the capsular reaction test for typing of pneumococci. J Clin Microbiol 1980;11:589-92.

${ }^{7}$ Trollfors B, Burman L, Dannetun E, Llompart J, Norrby R. Serotyping of Streptococcus pneumoniae strains by coagglutination and counterimmunoelectrophoresis. J Clin Microbiol 1983; 18:978-80.

${ }^{8}$ Lockley MR, Wise R. Pneumococcal infections. Br Med J 1984; 288:1179-80.

${ }^{9}$ Davies AJ, Hawkey PM, Simpson RA, O'Connor KM. Pneumococcal cross infection in hospital. Br Med J 1984;288:1195.

${ }^{10}$ Broome CV, Facklam RR, Fraser DW. Pneumococcal disease after pneumococcal vaccination: an alternative method to estimate the efficiency of pneumococcal vaccination. $N$ Engl J Med 1980;303:549-52.

${ }^{11}$ Smart LE, Girdwood RWA. Pneumococcal serotyping by direct examination of sputum samples for pneumococcal antigen. Comm Dis Scot 1982;82:5.

Requests for reprints to: Mr LE Smart, Department of Bacteriology, Stobhill Hospital, Glasgow G21 3UW, Scotland. 